\title{
Nano-Optics on Individual Quantum Objects - From Single to Coupled Semiconductor Quantum Dots
}

\author{
G. Bacher, H. Schömig, M.K. Welsch, M. Scheibner, \\ J. Seufert, M. Obert, A. Forchel \\ Technische Physik, Universität Würzburg \\ Am Hubland, 97074 Würzburg, Germany
}

\begin{abstract}
A.A. Maksimov, S. Zaitsev and V.D. Kulakovskit
Institute of Solid State Physics, RAS, 142432 Chernogolovka, Russia
\end{abstract}

\begin{abstract}
Some recent highlights of our optical studies on single and coupled semiconductor quantum dots are reviewed. In the first part, we concentrate on the role of spins and spin-spin interaction in nonmagnetic and magnetic single quantum dots. In the case of strictly resonant excitation of the ground state in self-assembled CdSe/ZnSe quantum dots, we find an exciton spin relaxation time, which exceeds the recombination lifetime significantly. Linear polarization has to be used for these experiments, as the electron-hole exchange interaction lifts the spin degeneracy and the eigenstates are linear combinations of spin-up and spin-down excitons. In a magnetic quantum dot, the exchange interaction between carrier spins and the spins of magnetic ions is shown to be responsible for giant magneto-optical effects. We demonstrate the formation of zero-dimensional magnetic polarons and we succeeded in measuring the magnetization on a scale of a few nanometers using the characteristic photoluminescence signal of individual quantum dots as experimental monitor. The second part is devoted to pairs of single quantum dots. On one hand, single exciton tunneling within an individual quantum dot pair is demonstrated studying single pairs of vertically correlated strain-induced and self-organized quantum dots. On the other hand, we show that in a pair of lithographically defined single dots with strongly different $g$-factors the energy spacing between the dot ground states can be tuned in an external magnetic field by about $10 \mathrm{meV}$, giving access to a controlled coupling between two individual quantum dots.
\end{abstract}

PACS numbers: 78.67.Hc, 75.50.Pp, 73.21.La 


\section{Introduction}

The huge interest in semiconductor quantum dots (QDs) is driven on one hand by a variety of promising applications making use of the characteristic $\delta$-like density of states and on the other hand by the possibility, to study fundamental physical aspects in these man-made "artificial atoms". The physical understanding of these quantum objects has significantly increased since researchers became able to study individual QDs, e.g. by means of spatially resolved photoluminescence (PL) spectroscopy [1-4]. Effects well known from atomic physics, like the Stark effect [5-9], the Zeeman effect [10-12], the Overhauser effect [13] and other more have been extensively investigated in single QDs during the last years. For example, it has been shown that applying external electric or magnetic fields can be used to manipulate the QD eigenstates in a well-defined manner. Moreover, the occupation of QD states with individual charge carriers can be controlled quite nicely. By varying the power of the exciting laser beam multiexciton formation has been observed $[10,11,14-16]$ and combining optical excitation with electrical carrier injection even allows to control the formation of charged excitons [17-19]. In contrast to "real" atoms, QDs offer the unique possibility to tailor the energy of the eigenstates, the $g$-factor of the charge carriers or even the magnetic properties just by varying the size and/or the composition of the QD. In particular, adding magnetic ions results in some characteristic magneto-optical effects, like exciton magnetic polaron formation [20,21] or giant $g$-factors [22] in quasi-zero-dimensional electronic systems. Going even a step further, some recent research efforts concentrate on fabricating and analyzing "artificial molecules" by combining two individual QDs with a well-defined spatial separation [23-26].

The large application potential of single and coupled QDs is evidenced by a variety of novel concepts envisioning devices used for single photon sources [27], single photon turnstiles [28], quantum dot memories [29] or even quantum computation [30, 31]. Loss and DiVincenzo [30], e.g., suggest to use the spin states of particles in a single QD as qubits. In that sense, the role of the spin of electrons, holes or excitons as well as the spin-spin interaction within a QD is of particular interest. Even more, as the spin relaxation time in QDs apparently exceeds the values found in higher dimensional systems significantly [32-34], the usage of (single) QDs should be also promising in the rapidly growing field of spintronics. One example of recent successes within this area is the application of (diluted) magnetic semiconductors (DMS) for spin injection into a nonmagnetic semiconductor [35-37]. Here, one might expect that combining the discrete density of states of zero-dimensional systems with the adjustable magnetic properties known from magnetic semiconductors will open a new path to devices based on the interaction between single carrier spins and the spins of magnetic ions. Quite recently, magnetic semiconductor QDs have been prepared by molecular beam epitaxy $[22,38-40]$ and first investigations on several interesting aspects, including 
$g$-factor engineering [22] and spin-spin interaction [20, 21, 41] have already been started.

In this contribution, we mainly focus on two aspects of QD physics. In the first part the spin relaxation and the spin-spin interaction in (single) semiconductor QDs is discussed. Both, the exchange interaction between charge carriers and its impact on the exciton spin relaxation, as well as the exchange interaction between carrier spins and the spins of magnetic ions, which allows to probe optically the QD magnetization with high spatial and temporal resolution, will be addressed. In the second part of the paper, we introduce two approaches for defining pairs of vertically correlated single QDs. This allows on one hand to demonstrate the (incoherent) tunneling of a single exciton from a strain-induced into a self-assembled QD and on the other hand to tune the energy spacing between the ground state of two adjacent, lithographically defined single QDs by about $10 \mathrm{meV}$ simply by applying an external magnetic field.

\section{Spins in nonmagnetic and magnetic quantum dots}

Both, the idea of using spin states as qubits in quantum computation [30] and the rapidly growing field of spintronics [42-44] raised a huge interest for a more detailed understanding of spin dynamics and spin-spin interaction in low dimensional semiconductors. In particular, the observation of long electron spin coherence times in $n$-doped bulk GaAs [45] or the experimental proof of enhanced exciton spin relaxation times in QDs as compared to higher dimensional system [32] can be regarded as important steps towards the realization of spin-based devices. Moreover, the growing interest of adding magnetic ions to semiconductors requires a deeper insight into the interaction between carrier spins and the spins of magnetic ions. Here, we discuss some important aspects of spin physics in low dimensional systems, in particular the spin relaxation in nonmagnetic QDs and the spin-spin interaction in magnetic semiconductor QDs.

\subsection{Exciton spin relaxation in CdSe/ZnSe quantum dots}

It is well known from transmission electron microscopy that in the CdSe/ZnSe system the QDs are more or less disk-shaped, i.e. the dot height is much smaller than its diameter $[4,46]$. Thus, we can characterize the spin state of the electron and the heavy hole by their components in growth direction, $s_{z}$ and $j_{z}$, respectively. The selection rules for optical transitions allow to address the spin states of electron-hole pairs quite easily by adjusting the polarization of the exciting laser beam. Only excitons, where the spins of the electron and the heavy hole are aligned antiparallel $\left(J_{z}=s_{z}+j_{z}= \pm 1\right)$ can directly couple to the light field. Exciting the QD ground state with $\sigma^{+}\left(\sigma^{-}\right)$polarized light should generate heavy hole excitons* with $J_{z}=1\left(J_{z}=-1\right)$. Monitoring the polarization degree as a function

*Due to strain and quantization effects, the light hole state is strongly shifted to higher energies as compared to the heavy hole state. 
of time will thus directly allow to extract the spin relaxation time of quasi-zero dimensional excitons.

We have investigated self-assembled CdSe/ZnSe QDs grown by migration enhanced epitaxy [47] on a GaAs substrate. A nominal CdSe thickness of 1.3 monolayers was used, which allows to tune the band gap of the QDs to about $2.6 \mathrm{eV}$. A frequency doubled, mode-locked Ti-sapphire laser (pulse width $1.5 \mathrm{ps}$ ), either linear or circular polarized was adjusted to the QD ground state. The transient photoluminescence (PL) signal of the photogenerated excitons was dispersed by a $0.46 \mathrm{~m}$ monochromator with a $300 \mathrm{~mm}^{-1}$ grating and detected by a Streak camera system with a temporal resolution of 20 ps. Strictly resonant excitation was used, i.e. the detection energy was identical to the excitation energy of $2.599 \mathrm{eV}$. All the measurements were done at $T=2 \mathrm{~K}$.

In Fig. 1, left, the PL signal after $\sigma^{+}$polarized excitation (i.e. only excitons with $J_{z}=+1$ are generated) is depicted for both, $\sigma^{+}$and $\sigma^{-}$polarized detection, respectively. No difference between these two traces is seen giving a time-independent polarization degree of $\rho_{\mathrm{cir}}=\left(I^{\sigma^{+}}-I^{\sigma^{-}}\right) /\left(I^{\sigma^{+}}+I^{\sigma^{-}}\right) \approx 0$. At first glance, this seems to indicate that the spin information transferred to the exciton by the exciting laser beam is completely lost within our time resolution. In contrast, if one excites with linear polarized light (i.e. $\pi^{x}$ and $\pi^{y}$, polarized along the [110] and the $[1 \overline{1} 0]$ crystal orientation, respectively), a strong difference is found, if one detects either the $\pi^{x}$ or the $\pi^{y}$ polarized PL signal (see right part of the figure). The PL signal is much larger, if the polarizations of the exciting laser pulse (in this case $\pi^{y}$ ) and the detected PL signal are identical. From the data we extract a polarization degree of $\rho_{\text {lin }}=\left(I^{\pi^{y}}-I^{\pi^{x}}\right) /\left(I^{\pi^{y}}+I^{\pi^{x}}\right) \approx 0.75$ and no decrease was found within the time window under investigation. This indicates that a once prepared exciton spin state is conserved at least for several nanoseconds, if one excites the QD ground state, in good agreement with recent results on self-assembled InAs/GaAs QDs [32].

In order to understand these results, i.e. the large and time-independent polarization degree after linear polarized excitation and the apparently ultrafast loss of spin information after circular polarized excitation, it is important to recall the influence of electron-hole exchange interaction on the symmetry of the eigenstates in self-assembled QDs. It has been shown that the spin degeneracy of the heavy hole exciton ground state is lifted due to exchange interaction $[3,10]$ and the fine structure splitting strongly depends on the symmetry of the QDs [10]. In fact, if the QD symmetry is e.g. reduced to $C_{2 v}$, the eigenstates of the bright excitons are given by

$$
\left|X^{1,2}\right\rangle=(|+1\rangle \pm|-1\rangle) / \sqrt{2} .
$$

As the exchange splitting of excitons in QDs is small as compared to the inhomogeneous broadening of an ensemble of QDs, one has to address single QDs in order to extract the fine structure and thus the symmetry of the eigenstates of zero-dimensional excitons. For selecting individual QDs, we have prepared small 


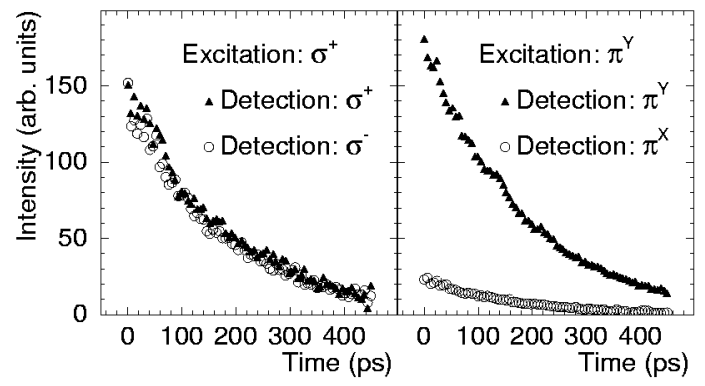

Fig. 1. PL intensity of the ground state emission of CdSe/ZnSe quantum dots versus time for different polarizations. The measurements have been performed at $T=2 \mathrm{~K}$. Left: circular polarized excitation and detection, right: linear polarized excitation/detection. Strictly resonant conditions have been applied, i.e. the excitation energy was identical to the detection energy.

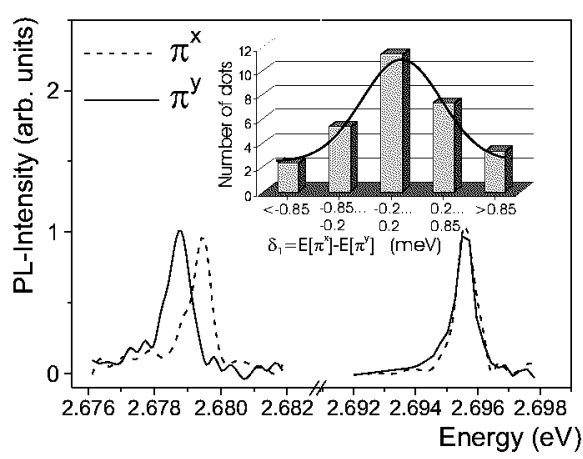

Fig. 2. PL spectra for two different CdSe/ZnSe single quantum dots obtained for $\pi^{x}$ and $\pi^{y}$ polarization, respectively. The inset shows a statistics of the exchange splitting $\delta_{1}$ between the ground state of the exciton obtained by comparing a variety of single quantum dots.

mesas with diameters down to $40 \mathrm{~nm}$ by electron beam lithography and wet chemical etching [4]. As the typical dot density is on the order of $10^{10}-10^{11} \mathrm{~cm}^{-2}$, one might expect only a few or even one single QD within such small mesas. In Fig. 2, we plotted linear polarized PL spectra of two individual CdSe/ZnSe QDs with similar growth parameters as the sample studied above. Low excitation density was used to ensure that only single electron-hole pairs occupy the QD ground state. While one QD $(E \approx 2.679 \mathrm{eV})$ shows a pronounced splitting between the $\pi^{x}$ and the $\pi^{y}$ polarized component of the PL spectrum, the energy splitting of these two components is negligible for the other one $(E \approx 2.6955 \mathrm{eV})$. As can be seen in the inset of the figure, the fine structure splitting varies from dot to dot and in some cases reaches values of up to $1 \mathrm{meV}$. Let us note that due to our finite spectral resolution of about $0.3 \mathrm{meV}$, small splittings cannot be resolved with our setup. 
The results obtained from time-resolved experiments and from spatially resolved measurements fit quite nicely together. As most of the QDs have at least a small asymmetry, the exciton eigenstates are predominantly linear combinations of $J_{z}=+1$ and $J_{z}=-1$. Thus, linear polarized excitation generates excitonic eigenstates. In consequence, the large time-independent polarization degree indicates a suppression of spin relaxation in QDs. In contrast, for circular polarized laser pulses, a coherent superposition of the exchange doublet (the band width of the laser pulse exceeds the energy splitting) is generated. However, as these experiments are performed on QD ensembles consisting of lots of dots with different exchange splittings, interference effects [48] prevent the observation of large polarization degrees or even photon beats, like observed by Flissikowskii et al. [49] on a single QD.

\subsection{Spin-spin interaction CdSe/ZnMnSe quantum dots}

In semimagnetic QDs like CdSe/ZnMnSe, exchange interaction does not only occur between electrons and holes, but in particular between carrier spins and the spins of magnetic ions in the crystal matrix. This has some important consequences, like the giant Zeeman effect or the formation of excitonic magnetic polarons (EMPs) [50-52]. An EMP is a small area in the crystal, where the spins of the charge carriers and the spins of the $\mathrm{Mn}^{2+}$ ions are strongly correlated due to $s p-d$ exchange interaction. In higher dimensional systems like bulk or quantum wells, the exciton wave function changes during the EMP formation due to (auto-)localization effects [53]. In contrast, in a wide-band gap self-assembled II-VI quantum dot (such as CdSe/ZnMnSe), the exciton is three-dimensionally confined. For magnetic fields below $\approx 10 \mathrm{~T}$, the dot radius is smaller than the typical magnetic length of $l=\sqrt{\hbar / e B}$. In addition, the energy spacing between the ground state and excited states is in the range of several tens of meV [54], i.e. much larger than the thermal energy for temperatures below $\approx 100 \mathrm{~K}$. Within these limits, neither increasing the temperature nor applying a magnetic field is expected to change the exciton wave function significantly and thus, the PL energy of a magnetic QD is expected to directly reflect changes in the magnetization via the $s p-d$ exchange interaction.

This can be nicely seen in time-resolved PL experiments, performed on self-assembled CdSe/ZnMnSe QDs grown by molecular beam epitaxy on a GaAs substrate. We chose a nominal CdSe thickness of 2.5 monolayers and a Mn concentration of $x=0.25$. In order to suppress nonradiative losses, we have taken care to prepare samples where the CdSe QD transition is energetically below the transition between internal $\mathrm{Mn}^{2+}$ states [38]. In Fig. 3, the transient energy shift of the PL signal of an ensemble of CdSe/ZnMnSe QDs after picosecond excitation is depicted. In the inset of the figure, PL spectra are shown for different delay times after the excitation pulse. 


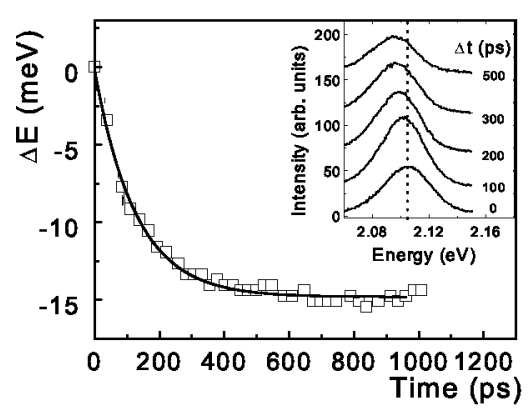

Fig. 3. Transient energy shift of the PL signal of self-assembled CdSe/ZnMnSe quantum dots after picosecond excitation. The solid line is a fit according to $\Delta E=-E_{\mathrm{MP}}\left(1-\mathrm{e}^{-t / \tau_{\mathrm{MP}}}\right)$. The inset shows transient PL spectra for different delay times $\Delta t$ after the excitation pulse. The measurements have been performed at $T=2 \mathrm{~K}$.

We found an energy shift of about $15 \mathrm{meV}$ during the first hundreds of picoseconds and a saturation of the transient shift after about half a nanosecond. This energy shift can be attributed to EMP formation: the Mn ion spins become aligned in the exchange field of the exciton and the reduction of the energy of the spin complex is seen as a red shift of the characteristic PL signal [41]. Several important points should be emphasized. First, the EMP formation time is found to be about $\tau_{\mathrm{MP}}=125 \mathrm{ps}$, i.e. much smaller than the recombination lifetime of $580 \mathrm{ps}$ in this sample. Thus, the whole EMP formation process until the equilibrium case, which is characterized by a complete alignment of the magnetic ion spins in the exciton exchange field, is monitored. Second, as the exciton wave function is not expected to change with time, the EMP formation time corresponds to the spin-spin scattering time, i.e. directly reflects the magnetization dynamics in magnetic semiconductor QD. In that sense, the EMP time constant may represent some high frequency limitation of potential devices based on the interaction between carrier spins and spins of magnetic ions in magnetic semiconductor quantum dots. $^{\dagger}$

The unique possibility to probe the magnetization via the optical response of magnetic semiconductor QDs offers an interesting chance: Addressing optically a single $\mathrm{CdSe} / \mathrm{ZnMnSe} \mathrm{QD}$ will give insight into magnetism on the nanometer scale! To get a sufficient spatial resolution for the selection of individual QDs metal apertures with diameters down to $80 \mathrm{~nm}$ have been defined lithographically on top of the samples [20,21]. The most straightforward way to influence the magnetization is to apply an external magnetic field and/or to change the temperature. In the left part of Fig. 4, spatially resolved PL spectra of single CdSe/ZnMnSe QDs are plotted for different magnetic fields. Low excitation density was used in order to ensure that only one single electron-hole pair was generated per QD. Surprisingly,

t The EMP formation time is of course expected to depend sensitively on parameters like the Mn-concentration, see T. Dietl et al. [53]. 
the individual emission peaks of the magnetic single QDs are characterized by a rather large line width of several meV, which is strongly reduced if one applies an external magnetic field in the Faraday geometry. In addition, a strong red shift occurs if one increases the magnetic field. This is the well-known giant Zeeman effect, a direct consequence of the $s p-d$ exchange interaction. Increasing the temperature on the other hand (see Fig. 4, right) causes a blue shift and a slight broadening of the individual PL peaks.

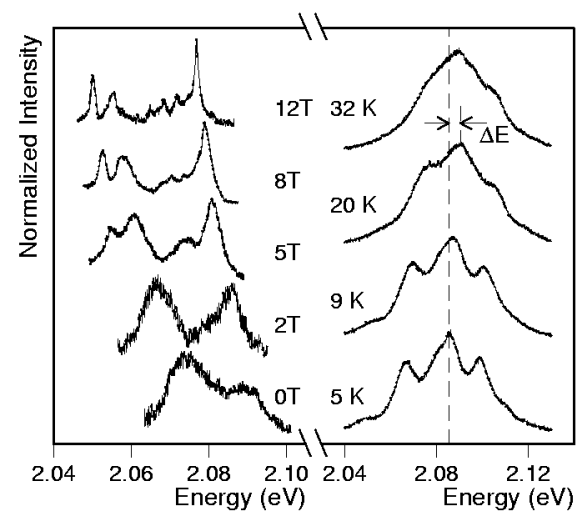

Fig. 4. Spatially resolved PL spectra of individual CdSe/ZnMnSe quantum dots for different external magnetic fields (left) and different temperatures (right). The data are obtained by using metal nanoapertures for single quantum dot selection with $\phi=250 \mathrm{~nm}$ and $\phi=175 \mathrm{~nm}$ in diameter, respectively.

In diluted magnetic semiconductors, the magnetization $M$ can be described by a modified Brillouin function [55]

$$
M(B, T)=x N_{0} g_{\mathrm{Mn}} \mu_{\mathrm{B}} S_{\mathrm{eff}} B_{5 / 2}\left(\frac{5 \mu_{\mathrm{B}} g_{\mathrm{Mn}} B}{2 k_{\mathrm{B}} T_{\mathrm{eff}}}\right),
$$

where $g_{\mathrm{Mn}}=2$ is the $g$-factor of $\mathrm{Mn}^{2+}$ ions, and $N_{0}$ is the number of cations per unit volume. The effective spin $S_{\text {eff }}<5 / 2$, and the effective temperature $T_{\text {eff }}=T+T_{0}$ take into account the antiferromagnetic interaction between neighboring $\mathrm{Mn}^{2+}$ spins [56]. The field $B=B_{\text {ext }}+B_{\text {MP }}$ includes both the external magnetic field $B_{\text {ext }}$ and the exchange field $B_{\mathrm{MP}}$ between the charge carriers and the spins of the magnetic ions $[21,57]$. Due to the $s p-d$ exchange interaction between the three-dimensionally confined carriers and the spins of the $\mathrm{Mn}^{2+}$ ions, the energy $E(B, T)$ of the $\mathrm{PL}$ signal from a single QD depends directly on the magnetization $M(B, T)$ within the exciton wave function. For example, for the $\sigma^{+}$-polarized component of the PL signal we can then write

$$
E(B, T)-E_{0}(T)=-\frac{g_{0} \mu_{\mathrm{B}} B_{\mathrm{ext}}}{2}-\frac{\gamma(\alpha-\beta)}{2 \mu_{\mathrm{B}} g_{\mathrm{Mn}}} M(B, T),
$$

where $\alpha$ and $\beta$ are the well-known exchange constants of the electrons and holes, respectively [58], $E_{0}(T)$ includes the temperature dependence of the band gap 
and $\gamma$ (which is less than unity) takes into account the fact that only a part of the exciton wave function actually overlaps with the $\mathrm{Mn}^{2+}$ ion spins [21]. If the Zeeman shift resulting from the band $g$-factor $g_{0}$ and the change of the band gap due to temperature are small, the PL energy of a single exciton directly monitors variations in the magnetization of the QD. ${ }^{\ddagger}$

In Fig. 5, we plotted the PL energy shift of a single QD versus magnetic field (a) and versus temperature (b). The symbols correspond to the experimental data, while the solid lines represent fits according to Eqs. (2) and (3). An excellent agreement between theory and experiment is obtained for both sets of data using $\gamma=0.34$ and an internal exchange field of $B_{\mathrm{MP}}=2.6 \mathrm{~T}$. This clearly confirms that our approach is well suited to monitor the magnetization on a scale defined by the extension of the exciton wave function.
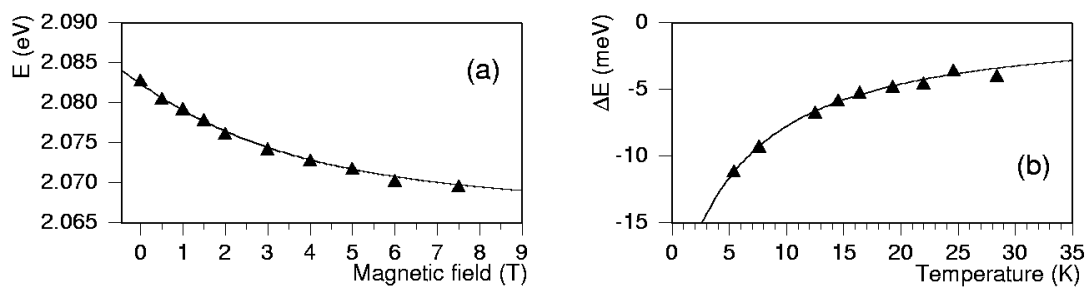

Fig. 5. Energy shift of an individual CdSe/ZnMnSe quantum dot versus magnetic field (a) and versus temperature (b). The temperature dependent data have been corrected by the (small) temperature dependent shift of the band gap. Symbols represent experimental data, the solid lines are fits according to Eq. (3).

At first glance one would expect that in the equilibrium case the EMP in a given single QD is characterized by a well-defined energy - i.e., the PL signal should be spectrally quite narrow. However, as the single QD is being probed repeatedly in our experiment, statistical variations of the orientation of the magnetic ion spins, i.e., fluctuations of the magnetization within the exciton wave function result in a broadening of the single QD emission peak in time-integrated experiments $[59,60]$. This is quite similar to what is known from fluctuating charges in the environment of a nonmagnetic single QD, which is found to result in a distinct line width broadening in cw PL measurements [61-64]. Intuitively it is clear that applying a magnetic field in the Faraday geometry will suppress the fluctuations of the magnetization explaining the line width narrowing seen in the experiment (see Fig. 4). It should be noted that such statistical magnetic fluctuations may be a severe limitation for the functionality of devices based on magnetic semiconductor QDs or other kinds of nanomagnetic systems.

¥This assumption is fulfilled in our case because of the large Mn-concentration, i.e., the large effective $g$-factor, and the small temperature range under investigation. 


\section{Approaches to pairs of single quantum dots}

Approaches to (single) quantum dot pairs for applications like quantum computation or quantum dot memories have to fulfill several requirements. First, one should be able to control the interdot distance very precisely, as e.g. the tunneling between two adjacent QDs depends exponentially on the spatial separation between the dots. Second, it is desirable to be able to control the energy spacing between the dots e.g. by external fields, which will allow to control the coupling in a QD molecule. From transport measurements, resonant tunneling of single electrons through discrete energy states of individual electrostatically defined quantum dots is well known and by changing the applied voltage, even the tunneling process can be controlled [65]. In contrast, only a few, quite recent publications deal with epitaxially defined single dot pairs. Incoherent tunneling between individual QDs have been reported quite recently by Seufert et al. [25] and Shtrichman et al. [26]. On the other hand, Schedelbeck et al. [23] and Bayer et al. [24] observed bound and antibound states in single quantum dot pairs prepared by cleaved edge overgrowth and self-organized growth, respectively. Although Shtrichman et al. have succeeded in applying an external electric field to a single pair of InAs/GaAs QDs, controlling the (coherent) tunneling of electrons, holes or excitons between two QDs still remains a challenge. Here, we discuss some of our recent results on single QD pairs with a well-defined vertical correlation between the dots. In particular, incoherent single exciton tunneling between a single pair of QDs is described and a new approach for defining QD molecules with adjustable energy spacing is presented.

\subsection{Single exciton tunneling between two correlated quantum dots}

For studying the (incoherent) tunneling of a single exciton between two correlated single QDs we follow an approach first introduced by Nikitin et al. [66]: Self-assembled CdSe QDs induce zero-dimensional states in a neighboring CdZnSe quantum well (QW) layer due to their strain and/or piezoelectric [67] field. The samples have been grown by migration enhanced epitaxy on [100] GaAs substrates. After a $200 \mathrm{~nm}$ GaAs buffer, a $50 \mathrm{~nm} \mathrm{ZnSe}$ layer followed by a CdZnSe QW with a well width of $10.5 \mathrm{~nm}$ and a $\mathrm{Cd}$ concentration of $x=0.08$ was deposited. Above the QW a ZnSe spacer layer of thickness $d(d=2.8 \mathrm{~nm}, 9 \mathrm{~nm}$ and $20 \mathrm{~nm}$, respectively) was grown followed by the deposition of 2.5 monolayers CdSe which leads to the formation of self-assembled QDs. A $25 \mathrm{~nm}$ ZnSe capping layer is used on top of the sample. As a reference, a QW sample without self-assembled QDs was grown. Using time-resolved PL spectroscopy on QD ensembles, an efficient tunneling between the CdZnSe and the CdSe layers was observed strongly depending on the spacer thickness. For example for a spacer thickness of $9 \mathrm{~nm}$ a tunneling time of about $40 \mathrm{ps}$ was found while the tunneling time decreases to $\leq 5 \mathrm{ps}$ in case of $d=2.8 \mathrm{~nm}$. 
In order to prove the formation of strain-induced QDs within the CdZnSe layer, spatially resolved PL spectroscopy was performed. The spatial resolution was obtained by etching small mesas with diameters down to $40 \mathrm{~nm}$ (see Sec. 2.1). For the sample with $d=9 \mathrm{~nm}$ and the reference QW, PL spectra obtained with different spatial resolution are plotted in Fig. 6. For decreasing mesa diameter, i.e. increasing lateral resolution the inhomogeneously broadened PL signal of the self-assembled CdSe QDs breaks up into several spectrally narrow lines, each line corresponding to the recombination of an exciton in an individual QD [4]. The same behavior is observed for the PL signal of the adjacent CdZnSe layer: here the narrow lines are attributed to excitons three-dimensionally localized in strain-induced QDs. In contrast, the PL spectrum of the reference QW (see Fig. 6, right) did not show any signature of quasi-zero-dimensional states even for the highest spatial resolution used $(40 \mathrm{~nm})$. Therefore we conclude that the strain field of the self-assembled QDs locally modulates the potential landscape of the CdZnSe layer forming strain-induced QDs.
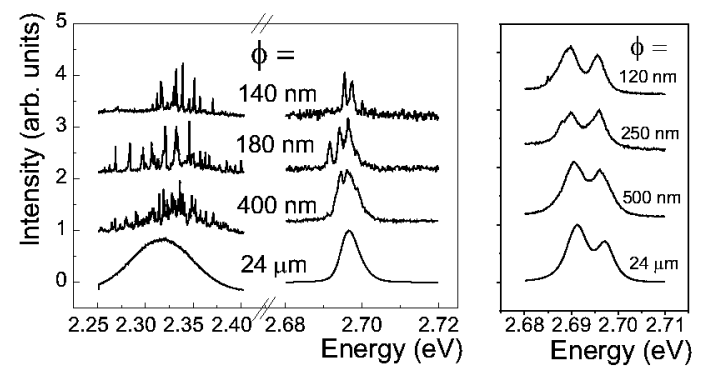

Fig. 6. PL spectra of the coupled quantum dot system obtained with different mesa sizes, i.e. different spatial resolutions. The spectral features centered at about $2.32 \mathrm{eV}$ arise from the self-assembled quantum dots, the $\mathrm{PL}$ signal at $\approx 2.7 \mathrm{eV}$ from the strain-induced quantum dots. For comparison, spatially resolved PL spectra for the CdZnSe quantum well reference sample are shown in the right part of the figure.

The possibility to access experimentally single pairs of self-assembled and strain-induced QDs allows us to investigate single exciton tunneling between adjacent QDs with a well-defined spatial separation. Experimentally, we applied the technique of photoluminescence excitation (PLE) spectroscopy: The detection energy was adjusted to the ground state emission of the self-assembled QDs, while the excitation energy was tuned across the band gap of the strain-induced QDs. In Fig. 7, the PLE spectrum obtained for ensembles of QD pairs (top) is compared to a PLE spectrum measured on a single QD pair (bottom). In addition, the corresponding PL spectra of the self-assembled QDs, centered around $2.32 \mathrm{eV}$ are depicted. The detection energy used for the PLE measurements is marked by an arrow. 


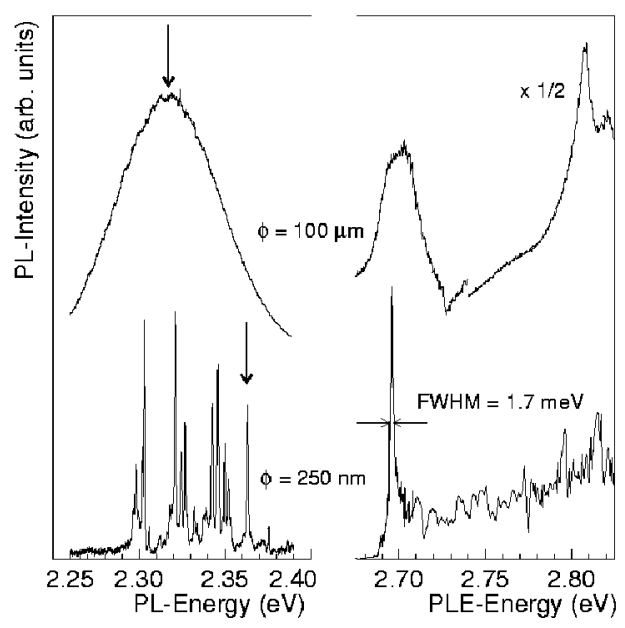

Fig. 7. PLE spectra for the coupled self-assembled - strain-induced quantum dot system for different spatial resolutions ( $\phi=100 \mu \mathrm{m}$ and $\phi=250 \mathrm{~nm}$, respectively). The detection energy was adjusted to the ground state of the self-assembled quantum dots while the excitation energy was tuned across the band gap of the strain-induced quantum dots. In addition, the corresponding PL spectra in the energy range of the self-assembled quantum dots are plotted. The arrows mark the detection energy used for measuring the PLE spectra.

In case of the ensemble measurements, the PLE resonance in the energy range of the ground state of the strain-induced QDs $(\approx 2.7 \mathrm{eV})$ is rather broad (FWHM $\approx 19.5 \mathrm{meV}$ ) due to inhomogeneous broadening effects. In contrast, performing the measurements on a single QD, a spectrally quite narrow PLE resonance at around $2.695 \mathrm{eV}$ (FWHM $\approx 1.7 \mathrm{meV}$ ) is found. This clearly demonstrates that a single exciton tunnels from a strain-induced QD to the corresponding energetically more favorable self-assembled QD [25, 54]. Out of the resonance the tunnel efficiency drops down drastically, indicating that we indeed observe an (incoherent) tunneling of a single zero-dimensional exciton between two correlated QDs. It should be noted that the energy of the PLE resonance varies from dot pair to dot pair due to size and/or composition variations of the individual QDs. The fact that both the emission line width of the single self-assembled QD (about $1 \mathrm{meV}$ ) and the line width of the PLE resonance is rather large may be due to the well-known effect of spectral diffusion [62].

\subsection{Correlated single dots with tunable energy spacing}

From an application point of view, it would be of great interest to tune the energy spacing between two adjacent quantum dots by an external field. This has been done by Shtrichman et al. [26], who applied a vertical electric field on a single pair of self-assembled quantum dots. However, fabricating stacks of self-assembled 
QDs with nominally identical ground state energy seems to be quite challenging and indeed, Shtrichman et al. observed an energetic difference of about $100 \mathrm{meV}$ between the ground states of the two single QD forming the QD molecule. For that reason, we chose a different approach for fabricating vertically correlated QDs: Starting from a double QW, where the thickness of both, the spacer and the QW can be controlled quite accurately, we define pairs of correlated QDs by electron beam lithography and selective intermixing.

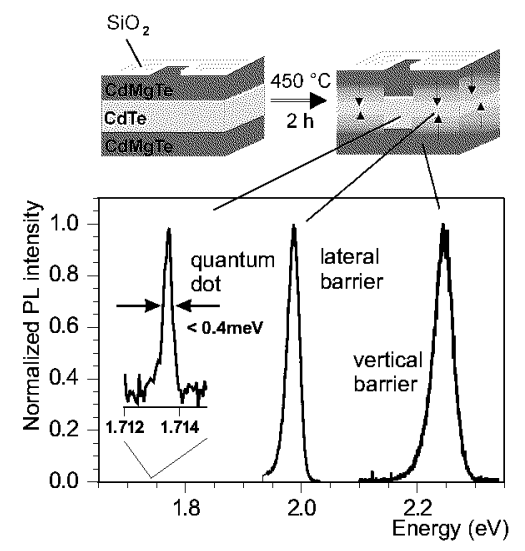

Fig. 8. Top: sketch of the principle idea of using selective interdiffusion to define buried single quantum dots. The small arrows shall indicate the diffusion of $\mathrm{Mg}$ atoms from the barrier to the quantum well region below the $\mathrm{SiO}_{2}$ cap layer. Bottom: representative PL spectrum of a single quantum dot defined with an $\mathrm{SiO}_{2}$ aperture diameter of $180 \mathrm{~nm}$.

In Fig. 8, the technique of selective intermixing for fabricating buried single QDs is schematically shown. An epitaxially grown $5 \mathrm{~nm}$ thick CdTe quantum well embedded between $\mathrm{Cd}_{1-x} \mathrm{Mg}_{x}$ Te barriers $(x=0.4)$ is covered by a $100 \mathrm{~nm}$ thick $\mathrm{SiO}_{2}$ layer which contains lithographically defined nanoapertures with diameters down to $80 \mathrm{~nm}$. A subsequent annealing step in an UHV chamber for 2 hours at $450^{\circ} \mathrm{C}$ causes an intermixing between the barrier and the well material, i.e. $\mathrm{Mg}$ atoms diffuse into the QW region. This intermixing process is strongly enhanced for areas covered by the $\mathrm{SiO}_{2}$ as compared to uncapped sample areas (like the regions of the nanoapertures) $[68,69]$. This allows a nice approach to define single QDs with adjustable size: $\mathrm{As}_{\mathrm{SiO}}$ increases the intermixing and thus the effective band gap of the quantum well layer, the capped areas act as lateral barriers after the annealing, while the carriers will be confined in the uncapped regions, i.e. the QDs. In fact, for the process parameters mentioned above, a lateral (exciton) confinement potential of about $0.27 \mathrm{eV}$ is realized. This is shown in Fig. 8, where the normalized PL spectrum of a lithographically defined single QD fabricated by using an aperture with a diameter of $180 \mathrm{~nm}$ is plotted. The PL spectrum consists of the emission from the single dot at about $1.7135 \mathrm{eV}$, from the lateral barrier at 
$\approx 1.98 \mathrm{eV}$ and from the vertical barrier at $2.25 \mathrm{eV}$. Let us note that due to the error function like lateral potential, the extension of the ground state exciton wave function is about $30 \mathrm{~nm}$, i.e. significantly smaller than the aperture size. Indeed, these QDs clearly demonstrate quasi 0D exciton properties, like e.g. line width narrowing down to $<0.4 \mathrm{meV}$ for an individual QD (see Fig. 8), the occurrence of biexcitons, and the recombination from excited states [68].

This technique to define buried single QDs based on ( $\mathrm{Cd}, \mathrm{Mg})$ Te heterostructures offers a variety of interesting opportunities. In particular, the effective $g$-factor in CdTe-based quantum wells can be tuned over a wide range simply by adding $\mathrm{Mn}$ atoms to the crystal matrix [50]. This is shown in Fig. 9, where the energy shift of the $\sigma^{+}$polarized component of the PL signal for thin CdMnMgTe/CdMgTe quantum wells is plotted for different $\mathrm{Mn}$ concentrations $x_{\mathrm{Mn}}$ versus magnetic field. A pronounced red shift of the PL signal due to the giant Zeeman effect is observed with increasing field, strongly depending on the Mn concentration $x_{\mathrm{Mn}}$.

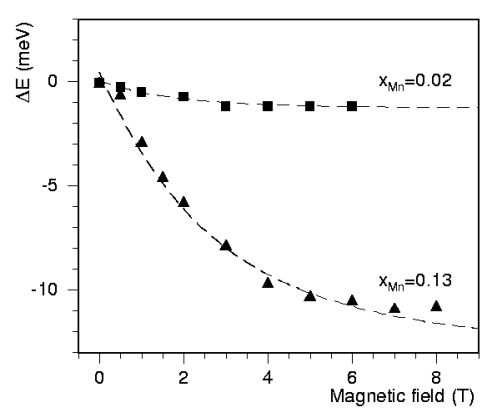

Fig. 9. Energy shift of the $\sigma^{+}$polarized component of the PL spectrum of $\mathrm{CdMnMgTe} / \mathrm{CdMgTe}$ quantum wells with different Mn concentrations $\left(x_{\mathrm{Mn}}=0.02\right.$ and $x_{\mathrm{Mn}}=0.13$, respectively) in an external magnetic field. The well width was $L_{z}=1 \mathrm{~nm}$ and the $\mathrm{Mg}$ concentration in the well $x_{\mathrm{Mg}}=0.05$ and in the barrier $x_{\mathrm{Mg}}=0.4$, respectively. The distinct change of the sensitivity of the band gap to the external magnetic field is obviously related to a strong dependence of the effective $g$-factor on the $\mathrm{Mn}$ concentration.

It is straightforward to apply our technique for single QD definition to a double QW system: Processing a double QW will result in a single pair of QDs with a well-defined spatial separation. Most interesting, choosing two QWs with different effective $g$-factors, one should be able to define pairs of single QDs with an energy spacing tunable by applying an external magnetic field. We used $\mathrm{CdMgTe} / \mathrm{CdTe} / \mathrm{CdMgTe} / \mathrm{CdTe} / \mathrm{CdMnTe}$ double QWs grown by molecular beam epitaxy on a CdZnTe substrate as a base material. The CdTe well width was $L_{z}=6 \mathrm{~nm}$, the CdMgTe spacer thickness $d=9 \mathrm{~nm}$ and $x_{\mathrm{Mn}}=x_{\mathrm{Mg}}=0.25$. Let us note that as the band gap energy CdMnTe and CdMgTe is comparable, we expect that the ground state energies of both QWs do not deviate very much from each 
other. However, due to the spatial overlap of the exciton wave function with the barriers and, in particular, due to a noticeable diffusion of Mn atoms in one (and only one!) of the QWs during QD fabrication, we can expect that the effective $g$-factors are strongly different for the two QDs forming the QD molecule.

Using essentially the same technology as described above, we have fabricated single pairs of coupled QDs. For the fabrication of the QD pair, we use an aperture with $\phi=270 \mathrm{~nm}$, which results in an extension of the ground state wave function of about $45 \mathrm{~nm}$, i.e. we are in the regime of center of mass quantization [68]. The PL spectrum of a single QD pair consists of the PL signal from the vertical barrier, the lateral barrier, and the PL signal resulting from the recombination of single excitons confined in the QDs. In Fig. 10, the Zeeman shift of the $\sigma^{+}$polarized component of the PL signal arising from the single QD pair is depicted versus magnetic field. Most important, a strong difference in the Zeeman shift of the two QDs is observed. While QD2 (embedded between one magnetic CdMnTe and one nonmagnetic CdMgTe barrier) strongly shifts to the red with increasing magnetic field, QD1 (embedded between two CdMgTe barriers) reveals only a weak energy shift, as expected for nonmagnetic QDs. This apparently originates from strongly different effective $g$-factors in the two QDs forming the molecule.

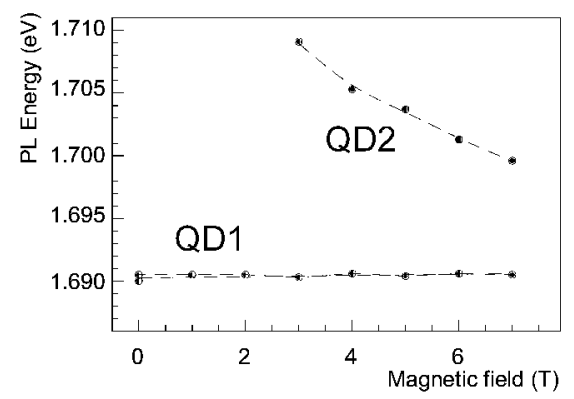

Fig. 10. Energy shift of the $\sigma^{+}$polarized component of the PL spectrum of a single lithographically defined quantum dot pair as a function of an external magnetic field in the Faraday geometry. The two dots within the quantum dot pair apparently have a strongly different effective $g$-factor, which allows to vary the energy spacing between the quantum dot ground states by about $10 \mathrm{meV}$.

It should be noted that at zero magnetic field a strong, incoherent tunneling from QD2 into QD1 prevents the observation of the PL signal from QD2. This tunneling probability is reduced, if the energy spacing between the dot ground states decreases and indeed the PL intensity ratio between QD2 and QD1 strongly increases with magnetic field. In fact, we have fabricated a single QD molecule containing vertically correlated QDs with well-defined spatial separation and strongly different effective $g$-factors. By applying an external magnetic field in the Faraday geometry, the relative energy spacing between the two QDs within the molecule 
can be varied by about $10 \mathrm{meV}$. Let us note that due to the large spacer thickness of $d=9 \mathrm{~nm}$ no anticrossing between the dot states is expected. However, as the spacer thickness $d$ can be controlled very accurately, our approach seems to be promising for fabricating single pairs of coherently coupled QDs, where the coupling can be adjusted by applying an external magnetic field.

\section{Summary}

Spatially resolved photoluminescence spectroscopy is applied to investigate both single quantum dots and single pairs of quantum dots. The spatial resolution is on one hand achieved lithographically by fabricating small mesas or small apertures to select individual quantum dots from epitaxially grown dot ensembles based on ZnSe compounds. On the other hand, we used electron beam lithography and selective intermixing to define single quantum dots and pairs of single quantum dots based on the CdTe system.

Several aspects which may be important for spin-based devices or quantum information processing have been addressed: Surprisingly large spin relaxation times at least in the nanosecond range of ground state excitons have been found in self-assembled CdSe/ZnSe quantum dots. Interestingly, such large spin relaxation times can only be observed, if one excites resonantly the exciton eigenstates, i.e. linear superpositions of $J_{z}=+1$ and $J_{z}=-1$ excitons. Adding magnetic ions to the crystal matrix is demonstrated to result in the formation of quasi-zero-dimensional exciton magnetic polarons. We have been able to show that due to the $s p-d$ exchange interaction between the carrier spins and the spins of the $\mathrm{Mn}^{2+}$ ions, the magnetization of a single magnetic semiconductor quantum dot can directly be monitored on a nanometer scale via the characteristic PL signal of the quantum dot.

In order to fabricate single pairs of correlated quantum dots, two approaches have been introduced. First, we used the strain field of self-assembled CdSe/ZnSe quantum dots to induce zero-dimensional states in an adjacent CdZnSe quantum well. The formation of strain-induced quantum dots is evidenced by spatially resolved photoluminescence spectroscopy and we have been able to observe (incoherent) single exciton tunneling between a single pair of quantum dots. Second, the technique of selective intermixing is used to fabricate both single quantum dots as well as single pairs of quantum dots based on CdTe. Most interesting, we have been able to show that two coupled quantum dots with strongly different effective $g$-factors can be prepared. This allows us to tune the energy spacing between the dot ground state in a single quantum dot pair by an external magnetic field by about $10 \mathrm{meV}$. 


\section{Acknowledgments}

It is our pleasure to thank M. Emmerling for expert technical assistance and P.S. Dorozhkin and A.V. Chernenko for experimental support. The epitaxial growth of the Cd(Zn)Se/ZnSe samples by K. Leonardi, Th. Passow and D. Hommel at Bremen University, the growth of the CdSe/ZnMnSe quantum dots by S. Lee, M. Dobrowolska and J.K. Furdyna at the University of Notre Dame and the growth of the CdTe/Cd(Mn,Mg)Te quantum wells by C.R. Becker and L.W. Molenkamp at Würzburg University is gratefully acknowledged. Financial support is obtained by the Deutsche Forschungsgemeinschaft (Ba 1422-1 and SFB 410) and the DARPA program.

\section{References}

[1] A. Zrenner, L.V. Butov, M. Hagn, G. Abstreiter, G. Böhm, G. Weimann, Phys. Rev. Lett. 72, 3382 (1994).

[2] H.F. Hess, E. Betzig, T.D. Harris, L.N. Pfeiffer, K.W. West, Science 264, 1740 (1994).

[3] D. Gammon, E.S. Snow, B.V. Shanabrook, D.S. Katzer, D. Park, Phys. Rev. Lett. 76, 3005 (1996).

[4] T. Kümmell, R. Weigand, G. Bacher, A. Forchel, K. Leonardi, D. Hommel, H. Selke, Appl. Phys. Lett. 73, 3105 (1998).

[5] S.A. Empedocles, M.G. Bawendi, Science 278, 2114 (1997).

[6] W. Heller, U. Bockelmann, G. Abstreiter, Phys. Rev. B 57, 6270 (1998).

[7] S. Raymond, J.P. Reynolds, J.L. Merz, S. Fafard, Y. Feng, S. Charbonneau, Phys. Rev. B 58, R13415 (1998).

[8] H. Gotoh, H. Kamada, H. Ando, J. Temmyo, Appl. Phys. Lett. 76, 867 (2000).

[9] J. Seufert, M. Obert, M. Scheibner, N.A. Gippius, G. Bacher, A. Forchel, T. Passow, K. Leonardi, D. Hommel, Appl. Phys. Lett. 79, 1033 (2001).

[10] V.D. Kulakovskii, G. Bacher, R. Weigand, T. Kümmell, A. Forchel, E. Borovitskaya, K. Leonardi, D. Hommel, Phys. Rev. Lett. 81, 1780 (1999).

[11] A. Zrenner, F. Findeis, E. Beham, M. Markmann, G. Böhm, G. Abstreiter, in: Advances in Solid State Physics 40, Ed. B. Kramer, Vieweg, Braunschweig/Wiesbaden 2000, p. 561.

[12] A. Kuther, M. Bayer, A. Forchel, A. Gorbunov, V.B. Timofeev, F. Schäfer, J.P. Reithmaier, Phys. Rev. B 58, R7508 (1998).

[13] S.W. Brown, T.A. Kennedy, D. Gammon, E.S. Snow, Phys. Rev. B 54, 17339 (1996)

[14] L. Landin, M.S. Miller, M.-E. Pistol, C.E. Pryor, L. Samuelson, Science 280, 262 (1998).

[15] G. Bacher, R. Weigand, J. Seufert, V.D. Kulakovskii, N.A. Gippius, A. Forchel, K. Leonardi, D. Hommel, Phys. Rev. Lett. 83, 4417 (1999).

[16] M. Bayer, O. Stern, P. Hawrylak, S. Fafard, A. Forchel, Nature 405, 923 (2000). 
[17] R.J. Warburton, C. Schäflein, D. Haft, F. Bickel, A. Lorke, K. Karrai, J.M. Garcia, W. Schoenfeld, P.M. Petroff, Nature 405, 926 (2000).

[18] F. Findeis, M. Baier, A. Zrenner, M. Bichler, G. Abstreiter, U. Hohenester, E. Molinari, Phys. Rev. B 63, 121309(R) (2001).

[19] G. Bacher, J. Seufert, M.K. Welsch, H. Schömig, M. Obert, V.D. Kulakovskii, A. Forchel, in: Advances in Solid State Physics 41, Ed. B. Kramer, Vieweg, Braunschweig/Wiesbaden 2001, p. 51.

[20] G. Bacher, H. Schömig, M.K. Welsch, S. Zaitsev, V.D. Kulakovskii, A. Forchel, S. Lee, M. Dobrowolska, J.K. Furdyna, B. König, W. Ossau, Appl. Phys. Lett. 79, 524 (2001).

[21] A.A. Maksimov, G. Bacher, A. McDonald, V.D. Kulakovskii, A. Forchel, C.R. Becker, G. Landwehr, L.W. Molenkamp, Phys. Rev. B 62, R7387 (2000).

[22] P.R. Kratzert, J. Puls, M. Rabe, F. Henneberger, Appl. Phys. Lett. 79, 2814 (2001).

[23] G. Schedelbeck, W. Wegscheider, M. Bichler, G. Abstreiter, Science 278, 1792 (1997).

[24] M. Bayer, P. Hawrylak, K. Hinzer, S. Fafard, M. Korkusinski, Z.R. Wasilewski, O. Stern, A. Forchel, Science 291, 451 (2001).

[25] J. Seufert, M. Obert, G. Bacher, A. Forchel, T. Passow, K. Leonardi, D. Hommel, Phys. Rev. B 64, 121303(R) (2001).

[26] I. Shtrichman, C. Metzner, B.D. Gerardot, W.V. Schoenfeld, P.M. Petroff, Phys. Rev. B 65, 081303(R) (2002).

[27] J.M. Gerard, B. Sermage, B. Gayral, B. Legrand, E. Costard, V. Thierry-Mieg, Phys. Rev. Lett. 81, 1110 (1998).

[28] P. Michler, A. Kiraz, C. Becher, W.V. Schoenfeld, P.M. Petroff, Lidong Zhang, E. Hu, A. Imamoglu, Science 290, 2282 (2000).

[29] T. Lundstrom, W. Schoenfeld, H. Lee, P.M. Petroff, Science 286, 2313 (1999).

[30] D. Loss, D.P. DiVincenzo, Phys. Rev. A 57, 120 (1998).

[31] A. Imamoglu, D.D. Awschalom, G. Burkard, D.P. DiVincenzo, D. Loss, M. Shervin, A. Small, Phys. Rev. Lett. 83, 4204 (1999).

[32] M. Paillard, X. Marie, P. Renucci, T. Amand, A. Jbeli, J.M. Gerard, Phys. Rev. Lett. 86, 1634 (2001).

[33] J.A. Gupta, D.D. Awschalom, X. Peng, A.P. Alivisatos, Phys. Rev. B 59, R10421 (1999).

[34] H. Gotoh, H. Ando, H. Kamada, A. Chavez-Pirson, Appl. Phys. Lett. 72, 1341 (1998).

[35] M. Oestreich, J. Hübner, D. Hägele, P.J. Klar, W. Heimbrodt, W.W. Rühle, D.E. Ashenford, B. Lunn, Appl. Phys. Lett. 74, 1251 (1999).

[36] R. Fiederling, M. Keim, G. Reuscher, W. Ossau, G. Schmidt, A. Waag, L.W. Molenkamp, Nature 402, 787 (1999).

[37] Y. Ohno, D.K. Young, B. Beschoten, F. Matsukura, H. Ohno, D.D. Awschalom, Nature 402, 790 (1999). 
[38] C.S. Kim, M. Kim, S. Lee, J. Kossut, J.K. Furdyna, M. Dobrowolska, J. Cryst. Growth 214/215, 395 (2000).

[39] Y. Oka, J. Shen, K. Takabayashi, N. Takahashi, H. Mitsu, I. Souma, R. Pittini, J. Lumin. 83-84, 83 (1999).

[40] I.I. Reshina, S.V. Ivanov, D.N. Mirlin, A.A. Toropov, A. Waag, G. Landwehr, Phys. Rev. B 64, 035303 (2001).

[41] J. Seufert, G. Bacher, M. Scheibner, A. Forchel, S. Lee, M. Dobrowolska, J.K. Furdyna, Phys. Rev. Lett. 88, 027402 (2002).

[42] S.A. Wolf, D.D. Awschalom, R.A. Buhrman, J.M. Daughton, S. von Molnar, M.L. Roukes, A.Y. Chtchelkanova, D.M. Treger, Science 294, 1488 (2001).

[43] P. Ball, Nature 404, 918 (2000).

[44] D.D. Awschalom, J.M. Kikkawa, Physics Today 52, 33 (1999).

[45] J.M. Kikkawa, D.D. Awschalom, Phys. Rev. Lett. 80, 4313 (1998).

[46] N. Peranio, A. Rosenauer, D. Gerthsen, S.V. Sorokin, I.V. Sedova, S.V. Ivanov, Phys. Rev. B 61, 16015 (2000).

[47] D. Hommel, K. Leonardi, H. Heinke, H. Selke, K. Ohkawa, F. Gindele, U. Woggon, Phys. Status Solidi B 202, 835 (1997).

[48] H. Nickolaus, H.-J. Wünsche, F. Henneberger, Phys. Rev. Lett. 81, 2586 (1998).

[49] T. Flissikowski, A. Hundt, M. Lowisch, M. Rabe, F. Henneberger, Phys. Rev. Lett. 86, $3172(2001)$.

[50] J.K. Furdyna, J. Appl. Phys. 64, R29 (1988).

[51] P.A. Wolff, in: Diluted Magnetic Semiconductors, Eds. J.K. Furdyna, J. Kossut, Semiconductors and Semimetals, Vol. 25, Academic, New York 1988, p. 413.

[52] For a review on exciton magnetic polarons in different dimensions see, e.g., D.R. Yakovlev, K.V. Kavokin, Comments Condens. Mat. Phys. 18, 51 (1996).

[53] T. Dietl, P. Peyla, W. Grieshaber, Y. Merle d'Aubigné, Phys. Rev. Lett. 74, 474 (1995)

[54] M. Rambach, J. Seufert, M. Obert, G. Bacher, A. Forchel, K. Leonardi, T. Passow, D. Hommel, Phys. Status Solidi B 229, 503 (2002).

[55] J.A. Gaj, R. Planel, G. Fishman, Solid State Commun. 29, 435 (1979).

[56] $S_{\text {eff }}=0.41$ and $T_{0}=3.6 \mathrm{~K}$ are taken from J.M. Fatah, T. Piorek, P. Harrison, T. Stirner, W.E. Hagston, Phys. Rev. B 49, 10341 (1994) and P.J. Klar, D. Wolverson, J.J. Davies, W. Heimbrodt, M. Happ, Phys. Rev. B 57, 7103 (1998), respectively.

[57] I.A. Merkulov, K.V. Kavokin, Phys. Rev. B 52, 1751 (1995).

[58] A. Twardowski, M. von Ortenberg, M. Demianiuk, R. Pauthenet, Solid State Commun. 51, 849 (1984).

[59] T. Dietl, J. Magn. Magn. Mater. 38, 34 (1983).

[60] G. Bacher, A.A. Maksimov, H. Schömig, M.K. Welsch, V.D. Kulakovskii, P.S. Dorozhkin, A. Forchel, S. Lee, M. Dobrowolska, J.K. Furdyna, to be published.

[61] S.A. Empedocles, D.J. Norris, M.G. Bawendi, Phys. Rev. Lett. 77, 3873 (1996). 
[62] J. Seufert, R. Weigand, G. Bacher, T. Kümmell, A. Forchel, K. Leonardi, D. Hommel, Appl. Phys. Lett. 76, 1872 (2000).

[63] V. Türck, S. Rodt, R. Heitz, R. Engelhardt, U.W. Pohl, D. Bimberg, R. Steingrüber, Phys. Rev. B 61, 9944 (2000).

[64] H.D. Robinson, B.B. Goldberg, Phys. Rev. B 61, R5086 (2000).

[65] N.V. van der Vaart, S.F. Godijn, Y.V. Nazarov, C.J.P.M. Harmans, J.E. Mooij, L.W. Molenkamp, C.T. Foxon, Phys. Rev. Lett. 74, 4702 (1995).

[66] V. Nikitin, P.A. Crowell, J.A. Gupta, D.D. Awschalom, F. Flack, N. Samarth, Appl. Phys. Lett. 71, 1213 (1997).

[67] J.H. Davies, Appl. Phys. Lett. 75, 4142 (1999).

[68] S. Zaitsev, M.K. Welsch, H. Schömig, G. Bacher, V.D. Kulakovskii, A. Forchel, B. König, C.R. Becker, W. Ossau, L.W. Molenkamp, Semicond. Sci. Technol. 16, 631 (2001).

[69] M.K. Welsch, H. Schömig, M. Legge, G. Bacher, A. Forchel, B. König, C.R. Becker, W. Ossau, L.W. Molenkamp, Appl. Phys. Lett. 78, 2937 (2001). 